\title{
OPERANDS AND INSTANCES
}

\author{
PETER FRITZ \\ Australian Catholic University and University of Oslo
}

\begin{abstract}
Can conjunctive propositions be identical without their conjuncts being identical? Can universally quantified propositions be identical without their instances being identical? On a common conception of propositions, on which they inherit the logical structure of the sentences which express them, the answer is negative both times. Here, it will be shown that such a negative answer to both questions is inconsistent, assuming a standard type-theoretic formalization of theorizing about propositions. The result is not specific to conjunction and universal quantification, but applies to any binary operator and propositional quantifier. It is also shown that the result essentially arises out of giving a negative answer to both questions, as each negative answer is consistent by itself.
\end{abstract}

§1. Introduction. Propositions are often assumed to reflect the logical structure of the sentences used to express them. Consider by way of example the case of conjunctions. It is natural to suppose that from the proposition expressed by a conjunctive sentence $\varphi \wedge \psi$, one can recover the conjuncts, i.e., the propositions expressed by $\varphi$ and $\psi$. Similarly, it is natural to suppose that from the proposition expressed by a universally quantified sentence $\forall v \varphi$, one can recover the instances, i.e., the propositions expressed by $\varphi$ on the various possible assignments of values to the free variable $v$.

This paper investigates such views logically, by regimenting the relevant theses in a formal language which allows quantification over propositions and pluralities of propositions. In a standard classical proof system for this langauge, it will be shown that the two recovery principles just outlined are inconsistent. In fact, the result generalizes beyond conjunction and universal quantification: there cannot be any binary sentential operator $\circ$ and propositional quantifier $Q$ such that the operands of $\circ$ and the instances of $Q$ can always be recovered. Furthermore, it will be shown that this inconsistency is essentially a result of the combination of two such principles, as the relevant principles are individually consistent.

Section 2 introduces the background logic of propositional and plural propositional quantification. Section 3 discusses a number of principles of logical structure, many of which turn out to lead directly to inconsistency via a result by Russell [12, Appendix B] and Myhill [11], and singles out some more promising candidates including the recoverability of operands and instances. Section 4 shows that such recovery principles are jointly inconsistent, and section 5 shows that they are individually consistent. Section 6 considers a number of refinements of the inconsistency result, pertaining to weakenings of the background logic and the recovery principles. Section 7 concludes.

Received: July 2, 2020.

2020 Mathematics Subject Classification: 03B16.

Key words and phrases: structured propositions, logical structure, higher-order logic, propositional quantifiers.

(C) The Author(s), 2021. Published by Cambridge University Press on behalf of The Association for Symbolic Logic. This is an Open Access article, distributed under the terms of the Creative Commons Attribution licence (http://creativecommons.org/licenses/by/4.0), which permits unrestricted re-use, distribution, and reproduction in any medium, provided the original work is properly cited. 
§2. Logic. The language to be used starts from the language of propositional logic, the formulas of which are built up from propositional variables $p, q \ldots$ using the primitive Boolean connectives $\neg, \wedge$, and $\vee$. To this, quantifiers $\forall$ and $\exists$ binding propositional variables are added, as in, e.g., [6]. Finally, plural analogs to such quantifiers are added. Building on ideas by Boolos [2], first-order logic can be enriched by variables and quantifiers which capture English sentences such as "there are some sets". Similarly, one may introduce new plural propositional variables $p p, q q, \ldots$, which can be bound by quantifiers $\forall$ and $\exists$ as well, and which may be used in statements of the form $\varphi \prec p p$, expressing that $\varphi$ is one of $p p$. For a more detailed discussion of plural propositional quantification, see [8].

DEFINITION 1 . Let $L$ be a language based on countably infinitely many propositional variables and countably infinitely many plural propositional variables, with formulas defined by the following clauses:

(1) Every propositional variable is a formula.

(2) If $\varphi$ and $\psi$ are formulas, then $\neg \varphi, \varphi \wedge \psi$, and $\varphi \vee \psi$ are formulas.

(3) If $\varphi$ is a formula and $v$ is a variable (propositional or plural propositional), then $\forall v \varphi$ and $\exists v \varphi$ are formulas.

(4) If $\varphi$ is a formula and $p p$ is a plural propositional variable, then $\varphi \prec p p$ is a formula.

The following abbreviations will be used:

$$
\begin{array}{ll}
\varphi \rightarrow \psi:=\neg \varphi \vee \psi & \varphi \leftrightarrow \psi:=(\varphi \rightarrow \psi) \wedge(\psi \rightarrow \varphi) \\
\varphi=\psi:=\forall p p((\varphi \prec p p) \leftrightarrow(\psi \prec p p)) & p p=q q:=\forall r((r \prec p p) \leftrightarrow(r \prec q q)) .
\end{array}
$$

In the definition of $\varphi=\psi, p p$ is the first plural propositional variable not free in $\varphi$ or $\psi . \neq$ will be used to abbreviate a negated application of $=$, and similarly for $\nprec$. Note that it will not be assumed that, e.g., $=$ expresses identity, but only that $\varphi=\psi$ is materially equivalent to the claim that $\varphi$ is $\psi . \varphi[\varepsilon / v]$ will be written for the result of replacing every free occurrence of $v$ in $\varphi$ by $\varepsilon$, assuming that $\varepsilon$ is free for $v$ in $\varphi . \varphi(\varepsilon)$ is used for $\varphi[\varepsilon / v]$ given a contextually salient variable $v$. In order to limit the number of parentheses required, it will be assumed that unary connectives $(\neg, \forall$ and $\exists$ ) bind stronger than binary connectives, and that among the latter, $=$ and $\prec$ bind strongest, after which come $\wedge$ and $\vee$, and finally $\rightarrow$ and $\leftrightarrow$.

The proof system to be used consists of standard classical axioms and rules for Boolean connectives and quantifiers, plus two axioms governing plural propositional quantification: first, a plural comprehension principle PC stating that for every condition $\varphi$, there are the propositions satisfying $\varphi$, and second, an extensionality principle EXT according to which these propositions are those propositions only if what holds of the former holds of the latter. (The comprehension PC may appear

\begin{tabular}{|c|c|c|c|}
\hline (TAUT) & tautologies & $(\mathrm{MP})$ & $\varphi, \varphi \rightarrow \psi / \psi$ \\
\hline (UI) & $\forall v \varphi \rightarrow \varphi[\varepsilon / v]$ & (UG) & $\varphi \rightarrow \psi / \varphi \rightarrow \forall v \psi(v$ not free in $\varphi)$ \\
\hline (QD) & $\exists v \varphi \leftrightarrow \neg \forall v \neg \varphi$ & $(\mathrm{PC})$ & $\exists p p \forall p(p \prec p p \leftrightarrow \varphi)(p p$ not free in $\varphi)$ \\
\hline (ExT) & $p p=q q \rightarrow(\varphi(p$ & & \\
\hline
\end{tabular}
unduly strong; this concern will be addressed below.)

DEFINITION 2. Let $\vdash$ be the proof system in $L$ with the following axiom schemas and rules: 
For the statement of Exт, recall that $\varphi(p p)$ and $\varphi(q q)$ are $\varphi[p p / r r]$ and $\varphi[q q / r r]$, respectively, for some suitable variable $r r$. The principle could thus also be stated more explicitly as follows:

$$
\text { (Ехт) } p p=q q \rightarrow(\varphi[p p / r r] \rightarrow \varphi[q q / r r]) .
$$

All elementary principles of quantification and identity can be derived in $\vdash$, and this will be assumed in the following. Let $\forall(\varphi)$ be the result of prefixing $\varphi$ with universal quantifiers binding the free variables in $\varphi . \Gamma \vdash \varphi$ will be used to state that there are $\gamma_{0}, \ldots, \gamma_{n} \in \Gamma$ such that $\vdash \forall\left(\gamma_{0}\right) \wedge \cdots \wedge \forall\left(\gamma_{n}\right) \rightarrow \forall(\varphi)$. Thus, when evaluating the deductive relationships between various principles, any free variables are taken to be implicitly universally quantified. This convention is adopted just to allow for a briefer statement of the various principles to be considered. As a consequence, a formula such as $p$ is interchangeable, in the context of $\vdash$, with $\forall p(p)$. The latter is refutable, in the sense that $\vdash \neg \forall p(p)$. Thus $\Gamma$ can be said to be consistent if $\Gamma \nvdash p$.

As an illustration of the use of $\vdash$, the following lemma shows that any proposition is distinct from its negation. (In subsequent proofs, deductions will be indicated less formally.)

Lemma 3. $\vdash p \neq \neg p$.

Proof. By the following deduction, which uses standard inferences easily seen to be licensed by $\vdash$ :
(1) $\forall q(q \prec p p \leftrightarrow q) \rightarrow((p \prec p p \leftrightarrow p) \wedge(\neg p \prec p p \leftrightarrow \neg p))$
UI
(2) $(p \prec p p \leftrightarrow \neg p \prec p p) \rightarrow \neg \forall q(q \prec p p \leftrightarrow q)$
(3) $\forall p p(p \prec p p \leftrightarrow \neg p \prec p p) \rightarrow \forall p p \neg \forall q(q \prec p p \leftrightarrow q)$
2, UI, UG
(4) $\exists p p \forall q(q \prec p p \leftrightarrow q) \rightarrow p \neq \neg p$
3
(5) $p \neq \neg p$
PC, 4

The conception of plural quantification used here allows for empty pluralities, which is arguably at odds with English phrases such as "there are some propositions such that ..." For, consider the following instance of PC:

$$
\exists p p \forall p(p \prec p p \leftrightarrow p \wedge \neg p) .
$$

From this, one obtains $\exists p p \forall p(p \nprec p p)$. But it sounds odd to say that there are some propositions such that no proposition is one of them. There are a number of ways in which this concern may be addressed. First, following Burgess and Rosen [3, p. 155], one may read $\exists p p \varphi$ as stating that there are zero or more propositions such that $\varphi$, and $\forall p p$ analogously. Second, one may consider quantifiers binding variables like $p p$ to be only loosely modeled on English plural talk, with their meaning determined in part by stipulative logical principles among which one may include PC, as suggested by Fritz et al. [8, Section 5.2]. Third, one may restrict PC to conditions which are satisfied, and add the principle that any propositions have some proposition among them:

$$
\begin{aligned}
& \left(\mathrm{PC}^{\prime}\right) \exists p \varphi \rightarrow \exists p p \forall p(p \prec p p \leftrightarrow \varphi) \quad(p p \text { not free in } \varphi) \\
& (\mathrm{PE}) \exists p(p \prec p p) .
\end{aligned}
$$

Let $\vdash^{\prime}$ be the variant of $\vdash$ which replaces PC by $\mathrm{PC}^{\prime}$ and PE. All of the results in this paper can be carried out using $\vdash^{\prime}$ instead of $\vdash$. For example, the instance of PC appealed to in the proof of of Lemma 3 is easily obtained from PC $^{\prime}$ using $\exists q q$. 
Fourth and finally, one may rephrase the results obtained here in terms of higher-order quantifiers binding variables in the position of sentential operators instead of plural propositional quantifiers; such a setting will be discussed in Section 6.1.

§3. Logical structure. With the formal system in place, the idea that propositions inherit the logical structure of the sentences which express them can be formalized.

3.1. Operator principles. In the case of sentential operators, formalization is relatively straightforward. For example, in the case of conjunction, to say that the conjuncts can be recovered from any conjunction is to say that two conjunctive formulas $\varphi \wedge \varphi^{\prime}$ and $\psi \wedge \psi^{\prime}$ express the same proposition only if $\varphi$ and $\psi$ express the same proposition, and likewise for $\varphi^{\prime}$ and $\psi^{\prime}$. In general, such a principle can be stated for any $n$-ary sentential operator $\circ$ :

$$
\left(\mathrm{O}_{\circ}\right) \circ\left(p_{1}, \ldots, p_{n}\right)=\circ\left(q_{1}, \ldots, q_{n}\right) \rightarrow p_{1}=q_{1} \wedge \cdots \wedge p_{n}=q_{n} .
$$

Such principles are implicit in many discussions of structured propositions. Explicit formulations can be found in formal developments of such views, such as $[1$, p. 65 , Axioms 10 and 11] and [10, p. 183, Principle 7].

The schematic principle $\mathrm{O}_{\circ}$ cannot be instantiated for $\prec$, since this is not a sentential operator. But a straightforward analog can be stated as follows:

$$
\left(\mathrm{O}_{\prec}\right)(p \prec p p)=(q \prec q q) \rightarrow p=q \wedge p p=q q .
$$

The only remaining logical operators of $L$ are quantifiers. But before considering the question how to formulate principles of logical structure for quantifiers, it is worth noting straight away that $\mathrm{O}_{\prec}$ is inconsistent. This is an immediate corollary of the following (variant of a) result by Russell [12, Appendix B] and Myhill [1]:

THEOREM 4 (Russell-Myhill). For any formula $\varphi$,

$$
\vdash \exists p p \exists q q(\varphi(p p)=\varphi(q q) \wedge p p \neq q q) .
$$

Proof. By plural comprehension, there are $q q$ such that:

$$
\forall q(q \prec q q \leftrightarrow \exists p p(q \nprec p p \wedge \varphi(p p)=q)) .
$$

Assume for contradiction that $\varphi(q q) \nprec q q$. Then for all $p p, \varphi(p p)=\varphi(q q)$ only if $\varphi(q q) \prec p p$. Thus $\varphi(q q)=\varphi(q q)$ only if $\varphi(q q) \prec q q$, whence $\varphi(q q) \prec q q$. This contradicts the assumption to the contrary, so $\varphi(q q) \prec q q$. So there are $p p$ such that $\varphi(q q) \nprec p p$ and $\varphi(p p)=\varphi(q q)$. Since $\varphi(q q) \prec q q$, it follows that $p p \neq q q$.

COROllary $5 . \mathrm{O}_{\prec}$ is inconsistent.

Proof. By Theorem 4, for any $p$, there are $p p \neq q q$ such that $(p \prec p p)=(p \prec q q)$, which contradicts $\mathrm{O}_{\prec}$.

In the proof of Theorem 4, it is not obviously ruled out that there is no $q$ such that $\exists p p(q \nprec p p \wedge \varphi(p p)=q)$. To carry out the deduction in $\vdash^{\prime}$, an auxiliary argument has to be added for this special case: If there is no $q$ such that $\exists p p(q \nprec p p \wedge \varphi(p p)=q)$, then $\forall p p(\varphi(p p) \prec p p)$. Let $p p$ be all the propositions, and $q q$ the propositions identical to $\varphi(p p)$; the existence of both follows from $\mathrm{PC}^{\prime}$. Then $\varphi(q q) \prec q q$, whence $\varphi(p p)=$ $\varphi(q q)$. But since there are at least two propositions (one true and one false), $p p \neq q q$. 
3.2. Some inconsistent quantifier principles. Returning to the question of how to formulate logical structure principles for quantifiers, the most straightforward attempt treats a quantifier binding a particular variable as a unary sentential operator like negation. For example, the instances of such a principle for universal propositional quantifiers have the following form:

$$
\text { (A) } \forall p \varphi=\forall p \psi \rightarrow \varphi=\psi \text {. }
$$

A version of this principle is discussed by Church [4, p. 514], who formalizes Russell's theory of structured propositions in a simple type theory. (See axiom 11; axioms 8-10 are noteworthy as well in corresponding to $\mathrm{O}_{\circ}$.) According to Church's formulation, quantified propositions are identical only if corresponding instances are identical:

(C) $\forall p \varphi=\forall p \psi \rightarrow \forall p(\varphi=\psi)$.

These two principles are easily seen to be equivalent, in the sense that $\mathrm{A} \vdash \mathrm{C}$ and $\mathrm{C} \vdash \mathrm{A}$, via UG and UI.

However, as Church [4, p. 520] notes, these principles are also shown to be inconsistent by the Russell-Myhill theorem. They entail:

$$
\forall p(p \prec p p)=\forall p(p \prec q q) \rightarrow \forall p((p \prec p p)=(p \prec q q)) .
$$

Identical propositions are materially equivalent, so from this one obtains:

$$
\forall p(p \prec p p)=\forall p(p \prec q q) \rightarrow p p=q q .
$$

And this is inconsistent by Theorem 4 .

As is easily seen, this observation applies as well to existential quantifiers. A similar argument can also be given for plural propositional quantifiers. Consider the following instance of the analog of $\mathrm{C}$ for plural propositional quantifiers:

$$
\forall r r(r r=p p)=\forall r r(r r=q q) \rightarrow \forall r r((r r=p p)=(r r=q q))
$$

Instantiating the quantification of the consequent using $p p$, one obtains with the material equivalence of identicals and $p p=p p$ :

$$
\forall r r(r r=p p)=\forall r r(r r=q q) \rightarrow(p p=q q) .
$$

And this is again inconsistent by Theorem 4 .

The problem with principles A and C may appear to be the treatment of quantifiers as variable-binding operators. But the problem persists if one introduces a variable binding $\lambda$-operator, and treats quantifiers as higher-order predicates, as suggested, e.g., by Stalnaker [13]. For, consider an extension of $L$ in which for every propositional variable $p$ and formula $\varphi$, there is a unary sentential operator $\lambda p . \varphi$. Then $\forall p \varphi$ can be treated as an abbreviation for $\forall \lambda p . \varphi$, which suggests a principle of logical structure for the quantifiers with the following instances:

$$
(\forall \lambda p . \varphi)=(\forall \lambda p . \psi) \rightarrow(\lambda p . \varphi)=(\lambda p . \psi) .
$$

But this is again inconsistent, for much the same reasons as before. Consider the following instance:

$$
(\forall \lambda p \cdot p \prec p p)=(\forall \lambda p \cdot p \prec q q) \rightarrow(\lambda p \cdot p \prec p p)=(\lambda p \cdot p \prec q q) .
$$

By UG, the consequent can be universally quantified, the result of which entails $\forall p((\lambda p . p \prec p p) p \leftrightarrow(\lambda p . p \prec q q) p)$. With the elementary principle of extensional (or 
material) $\beta$-conversion, according to which $(\lambda p . \varphi) \psi$ is materially equivalent to $\varphi[\psi / p]$, this in turn entails $\forall p(p \prec p p \leftrightarrow p \prec q q)$. Thus, it follows:

$$
(\forall \lambda p \cdot p \prec p p)=(\forall \lambda p \cdot p \prec q q) \rightarrow p p=q q .
$$

And this was noted to be inconsistent by Theorem 4 .

3.3. Dispensing with order. Church's formulation $\mathrm{C}$ of the logical structure principle for universal quantifiers is interesting since it corresponds in a natural way to $\mathrm{O}_{\wedge}$. Consider the view on which universal and existential quantifiers serve to express long conjunctions and disjunctions, respectively. Roughly, this view holds that a universal quantification $\forall p \varphi(p)$ serves to express a conjunction of the form:

$$
\bigwedge \varphi\left(p_{0}\right) \varphi\left(p_{1}\right) \cdots,
$$

where the propositions expressed by $p_{0}, p_{1}, \ldots$ constitute a well-order of the propositions, and $\Lambda$ is an infinitary sentential operator which takes as arguments a sequence of formulas of the relevant order type. (Set aside worries about there being more propositions than propositional variables. The relevant extension of $L$ is only appealed to loosely to motivate principles which will themselves be stated in $L$.)

Assume now that $\forall p \varphi(p)=\forall p \psi(p)$. On the view under consideration, this can equivalently be stated as:

$$
\bigwedge \varphi\left(p_{0}\right) \varphi\left(p_{1}\right)=\bigwedge \psi\left(p_{0}\right) \psi\left(p_{1}\right) \cdots
$$

The relevant generalization of $\mathrm{O}_{\wedge}$ should allow us to obtain from this the following sequence of claims:

$$
\varphi\left(p_{0}\right)=\psi\left(p_{0}\right), \varphi\left(p_{1}\right)=\psi\left(p_{1}\right), \ldots
$$

But since $p_{0}, p_{1}, \ldots$ are assumed to comprise all propositions, this is equivalent to the claim that $\forall p(\varphi(p)=\psi(p))$, as Church's principle states.

This correspondence between the two principles is interesting, since it suggests a natural weakening of Church's principle $\mathrm{C}$. Notice that $\mathrm{O}_{\wedge}$ is immediately inconsistent with the commutativity of conjunction, i.e., the following principle:

$$
(p \wedge q)=(q \wedge p) \text {. }
$$

The inconsistency follows from the fact that commutativity entails $(p \wedge \neg p)=(\neg p \wedge$ $p$ ), which, with $\mathrm{O}_{\wedge}$, leads to the absurd $p=\neg p$.

But even someone who is attracted to propositions having some amount of logical structure might want to endorse the commutativity of conjunction (and analogously disjunction). As Dorr [5, p. 124, en. 56] notes, one might adapt an idea of Williamson [16, p. 259] and conceive of the formation of conjunctions as amounting to putting conjuncts into a conjunctive bag, from which conjuncts can be recovered, but not necessarily in order. This motivates the following weaker logical structure principle for binary sentential operators:

$$
\left(\mathrm{O}_{\circ}^{-}\right)\left(p \circ p^{\prime}\right)=\left(q \circ q^{\prime}\right) \rightarrow\left(p=q \wedge p^{\prime}=q^{\prime}\right) \vee\left(p=q^{\prime} \wedge p^{\prime}=q\right) .
$$

Returning to the case of quantifiers (illustrated using universal propositional quantifiers), one might correspondingly conceive of $\forall p \varphi(p)$ as serving to express a conjunction of the following form:

$$
\bigwedge\left\{\varphi\left(p_{0}\right), \varphi\left(p_{1}\right), \ldots\right\},
$$


where $\bigwedge$ is now understood as a unary operator taking a set of formulas as an argument. The idea that conjuncts can be recovered from conjunctions, but not necessarily in order, thus suggests that $\forall p \varphi(p)$ is only identical to $\forall p \psi(p)$ if for every index $i, \varphi\left(p_{i}\right)$ is $\psi\left(p_{j}\right)$, for some index $j$, and vice versa. In $L$, this can be stated more succinctly as follows:

$$
\forall p \varphi(p)=\forall p \psi(p) \rightarrow \forall p \exists q(\varphi(p)=\psi(q)) \wedge \forall p \exists q(\psi(p)=\varphi(q)) .
$$

Here, $q$ is the first variable free for $p$ in $\psi$ distinct from $p$. Note that since identity is symmetric, only one of the conjuncts in the consequent need be included in the formulation of this schematic principle. Thus, the following slightly simpler principle suffices:

$$
\left(\mathrm{I}_{\forall p}\right) \forall p \varphi(p)=\forall p \psi(p) \rightarrow \forall p \exists q(\varphi(p)=\psi(q)) .
$$

The analog of this principle for existential propositional quantifiers is the following:

$$
\left(\mathrm{I}_{\exists p}\right) \exists p \varphi(p)=\exists p \psi(p) \rightarrow \forall p \exists q(\varphi(p)=\psi(q)) .
$$

And, letting $Q$ be either $\forall$ or $\exists$, the analog of $\mathrm{I}_{Q p}$ for plural propositional quantifiers reads as follows:

$$
\left(\mathrm{I}_{Q p p}\right) Q p p \varphi(p p)=Q p p \psi(p p) \rightarrow \forall p p \exists q q(\varphi(p p)=\psi(q q)) .
$$

Stepping back from the view of quantifiers as serving to express long conjunctions and disjunctions, the weaker principles of $\mathrm{O}_{\circ}^{-}$and $\mathrm{I}_{Q v}$ (where $v$ may be a propositional or a plural propositional variable) encapsulate independently natural views of operators and quantifiers: operands and instances can always be recovered, but not necessarily in any particular order. And $\mathrm{I}_{Q v}$ does not appear to suffer from the same problem which plagued the inconsistent principles of logical structure for quantifiers considered in the previous section. For, consider the instance for the formulas which there led to inconsistency:

$$
\forall p(p \prec p p)=\forall p(p \prec q q) \rightarrow \forall p \exists q((p \prec p p)=(q \prec q q)) .
$$

Assume for the sake of the argument that any formula of the form $p \prec p p$ expresses one of two propositions $t$ and $f$ (the first being true and the second being false). With this, $\forall p \exists q((p \prec p p)=(q \prec q q))$ can be seen not to entail, in general, $p p=q q$ : For example, consider distinct pluralities of propositions $p p$ and $q q$, which both have some proposition among them, and both some proposition not among them. Such pluralities must exist; for example, $p p$ might be the truths and $q q$ the falsities. Then the instances of $\forall p(p \prec p p)$ will comprise $t$ and $f$, and so will the instances of $\forall p(p \prec q q)$.

3.4. Summary. The previous sections singled out the following principles, one for each logical connective, with the principles for binary sentential operators coming in an ordered and an unordered variant:

$$
\mathrm{O}_{\neg}, \mathrm{O}_{\wedge}^{(-)}, \mathrm{O}_{\vee}^{(-)}, \mathrm{I}_{\forall p}, \mathrm{I}_{\exists p}, \mathrm{I}_{\forall p p}, \mathrm{I}_{\exists p p}, \mathrm{O}_{\prec},
$$

$\mathrm{O}_{\prec}$ was shown to be inconsistent since it allows one to recover a plural propositional parameter, which the Russell-Myhill result shows to be impossible. But none of the the other principles obviously shares this feature, which poses the question which of them are individually and jointly consistent. Sections 4 and 5 answer this question.

Section 4 shows that if $\circ$ is a binary operator and $Q p$ is a propositional quantifier, then the corresponding instances of $\mathrm{O}_{\circ}^{-}$and $\mathrm{I}_{Q p}$ are jointly inconsistent; a fortiori, 
the corresponding instances of $\mathrm{O}_{\circ}$ and $\mathrm{I}_{Q p}$ are jointly inconsistent as well. Section 5 shows that these inconsistency results delineate precisely the consistent combinations of principles of logical structure. That is, it is there shown that following theories are consistent, and maximally so among sets of the principles considered here:

$$
\begin{aligned}
& T_{o}:=\left\{\mathrm{O}_{\neg}, \mathrm{O}_{\wedge}, \mathrm{O}_{\vee}, \mathrm{I}_{\forall p p}, \mathrm{I}_{\exists p p}\right\} \\
& T_{i}:=\left\{\mathrm{O}_{\neg}, \mathrm{I}_{\forall p}, \mathrm{I}_{\exists p}, \mathrm{I}_{\forall p p}, \mathrm{I}_{\exists p p}\right\} .
\end{aligned}
$$

§4. Inconsistency. The argument to be given is a variant of an argument which shows that natural principles of immediate grounding are inconsistent, and derived from [7]. The first step is to show how from any operator $\circ$ satisfying $\mathrm{O}_{\circ}^{-}$, an operator $\hat{o}$ can be defined which satisfies $\mathrm{O}_{\hat{o}}$ :

$$
\varphi \hat{\circ} \psi:=((\varphi \circ \neg \varphi) \circ(\varphi \circ \varphi)) \circ(\psi \circ \psi) \text {. }
$$

LEMMA $6 . \quad \mathrm{O}_{\circ}^{-} \vdash \mathrm{O}_{\hat{\circ}}$.

Proof. Assume $\left(p \hat{\circ} p^{\prime}\right)=\left(q \hat{\circ} q^{\prime}\right)$, i.e.:

(1) $\left(((p \circ \neg p) \circ(p \circ p)) \circ\left(p^{\prime} \circ p^{\prime}\right)\right)=\left(((q \circ \neg q) \circ(q \circ q)) \circ\left(q^{\prime} \circ q^{\prime}\right)\right)$.

The following uses $\mathrm{O}_{\circ}^{-}$. By Lemma 3, $p \neq \neg p$, whence $(p \circ \neg p) \neq(p \circ p)$. So:

(2) $((p \circ \neg p) \circ(p \circ p)) \neq\left(q^{\prime} \circ q^{\prime}\right)$

From (1) and (2), the following two claims can be inferred:

(3) $((p \circ \neg p) \circ(p \circ p))=((q \circ \neg q) \circ(q \circ q))$

(4) $\left(p^{\prime} \circ p^{\prime}\right)=\left(q^{\prime} \circ q^{\prime}\right)$

By $(3)$, since $(p \circ \neg p) \neq(q \circ q),(p \circ p)=(q \circ q)$, whence $p=q$. By $(4), p^{\prime}=q^{\prime}$.

Using this lemma, $\mathrm{O}_{\circ}^{-}$and $\mathrm{I}_{Q p}$ can be seen to entail that from $Q p(p \prec r r \hat{\circ} p)$, the $r r$ can be recovered, and by the Russell-Myhill theorem, this is inconsistent:

LEMma 7. For $Q$ being one of propositional quantifiers $\forall$ and $\exists$ :

$$
\mathrm{O}_{\circ}^{-}, \mathrm{I}_{Q p} \vdash Q p(p \prec p p \hat{\circ} p)=Q p(p \prec q q \hat{\circ} p) \rightarrow p p=q q
$$

Proof. Assume $Q p(p \prec p p \hat{\circ} p)=Q p(p \prec q q \hat{\circ} p)$. By $\mathrm{I}_{Q p}$, it follows that:

$$
\forall p \exists q((p \prec p p \hat{\circ} p)=(q \prec q q \hat{\circ} q)) .
$$

So by Lemma 6:

$$
\forall p \exists q((p \prec p p)=(q \prec q q) \wedge p=q) .
$$

Thus $\forall p((p \prec p p)=(p \prec q q))$, whence $p p=q q$.

THEOREM 8. If $Q p$ is a propositional quantifier and $\circ$ is a binary operator, then $\mathrm{O}_{\circ}^{-}$and $\mathrm{I}_{Q p}$ are jointly inconsistent.

Proof. Immediate by Lemma 7 and Theorem 4.

Note that while $L$ only provides two binary operators (conjunction and disjunction) and two propositional quantifiers (existential and universal), the argument for the inconsistency of $\mathrm{O}_{\circ}$ and $\mathrm{I}_{Q p}$ does not appeal to any of their particular features. Thus, Theorem 8 generalizes straightforwardly to any language with additional binary operators and propositional quantifiers. 
§5. Consistency. First, a class of models will be defined which is sufficiently general to provide models to prove both $T_{o}$ and $T_{i}$ consistent, after which more specific classes of models for each of the theories will be identified. These models are specifically tailored to these consistency results, and are not meant to capture any notion of validity which is of independent interest.

5.1. Models. Models will be based on a set $X$ of propositions. Each of these propositions will be associated with two items of information: first, its instances or operands (no distinction needs to be made between the two concepts), and second, its truth value, using 0 and 1 for falsity and truth, respectively. By Cantor's theorem, there are more sets of propositions than propositions, so only some sets of propositions will serve as the set of instances of some proposition. These sets will be called well-behaved. The set $W$ of well-behaved sets of propositions will be assumed to contain all finite sets. The correspondence between propositions and their instances and truth-values will be given by a bijection $c$ mapping every pair $\langle I, t\rangle$ consisting of a well-behaved set $I$ of instances and a truth-value $t$ to a proposition. Finally, a model will contain a switch $s$, which is $o$ or $i$ depending on whether the model is intended to validate $T_{o}$ or $T_{i}$.

Since $c$ is a bijection, the instances and truth-value of a proposition $x$ can be recovered as the first and second coordinate of $c^{-1}(x)$, which will be notated $\pi_{1} c^{-1}(x)$ and $\pi_{2} c^{-1}(x)$. For stating various definitions, it will be useful to extend $c$ to a function $\tilde{c}$ which applies to $\langle I, t\rangle$ for all $I \subseteq X$ and $t<2$. The particular choice of this extension is unimportant, but the simplest option is to let $\tilde{c}\langle I, t\rangle$ always be $c\langle\emptyset, t\rangle$ whenever $c\langle I, t\rangle$ is undefined.

Definition 9. A model is a tuple $\langle X, W, c, s\rangle$ such that:

$X$ is an infinite set.

$W \subseteq \mathcal{P}(X)$ such that $Y \in W$ for all finite sets $Y \subseteq X$.

$c: W \times\{0,1\} \rightarrow X$ is a bijection.

$s \in\{o, i\}$.

For any model, define, for all $x \in X$ :

$$
\begin{aligned}
& l(x)=\pi_{1} c^{-1}(x) \\
& \tau(x)=\pi_{2} c^{-1}(x) .
\end{aligned}
$$

Extend $c$ to a function $\tilde{c}: \mathcal{P}(X) \times\{0,1\} \rightarrow X$ by letting:

$$
\tilde{c}\langle I, t\rangle= \begin{cases}c\langle I, t\rangle & \text { if } I \in W, \\ c\langle\emptyset, t\rangle & \text { otherwise. }\end{cases}
$$

Assignment functions map propositional variables to members of $X$, and plural propositional variables to subsets of $X$. As usual, for any assignment function $a, a[\xi / v]$ is the function mapping $v$ to $\xi$ and every other variable $v^{\prime}$ to $a\left(v^{\prime}\right)$, and correspondingly for sequences of variables. Relative to an assignment function $a$, a model interprets any formula $\varphi$ using an element $\llbracket \varphi \rrbracket^{a}$. This can be specified by determining the instances (operands) and truth value separately. Consider, by way of illustration, the case of negation. To validate $\mathrm{O}_{\neg}$, negation should preserve instances (operands), but flip the truth value. Thus, the set of instances and the truth value of the proposition expressed by $\neg \varphi$ should be $l\left(\llbracket \varphi \rrbracket^{a}\right)$ and $1-\tau\left(\llbracket \varphi \rrbracket^{a}\right)$, respectively. Therefore, $\llbracket \neg \varphi \rrbracket^{a}$ can be specified as $\tilde{c}\left\langle\imath\left(\llbracket \varphi \rrbracket^{a}\right), 1-\tau\left(\llbracket \varphi \rrbracket^{a}\right)\right\rangle$. Note that for such a specification to have the intended effect, it is important that the relevant set of instances be well-behaved. In 
the case of negation, this is easily seen to be guaranteed, but in other cases, verifying this will be an important aspect in showing that the models validate the intended theories.

The other connectives are treated similarly, depending on the theory which is to be validated. For example, if $s=o$, then conjuncts should be recoverable from conjunctions, so the instances of the proposition expressed by a conjunction $\varphi \wedge \psi$ should be the propositions expressed by $\varphi$ and $\psi$. If $s=i$, then no particular constraint is imposed on conjunctions, so it may simply be stipulated that the set of instances is empty. Thus the set of instances of $\llbracket \varphi \wedge \psi \rrbracket^{a}$ can be specified as $\left\{\llbracket \varphi \rrbracket^{a}, \llbracket \psi \rrbracket^{a}: s=o\right\}$. Similarly, the set of instances of $\llbracket \forall p \varphi \rrbracket^{a}$ can be specified as the set of propositions $\llbracket \varphi \rrbracket^{a[x / p]}$ for $x \in X$, assuming $s=i$. Plural propositional quantifiers are treated similarly, independently of the choice of $s$. Finally, since neither $T_{o}$ nor $T_{i}$ impose any particular constraints on $\prec$, the set of instances of a proposition expressed by $\varphi \prec p p$ may always be assumed to be empty. The truth-value of this proposition is determined by whether $\llbracket \varphi \rrbracket^{a}$ is a member of $a(p p)$. Taking 0 (falsity) to be $\emptyset$ and 1 (truth) to be $\{\emptyset\}$, this may be specified as $\left\{\emptyset: \llbracket \varphi \rrbracket^{a} \in a(p p)\right\}$.

Truth of a formula $\varphi$ relative to a model and assignment function $a$ is determined by $\tau\left(\llbracket \varphi \rrbracket^{a}\right)$, and validity is defined as truth relative to every assignment function and model.

DEFINITION 10. An assignment function for a model is a function $a$ such that $a(p) \in X$ for every propositional variable $p$, and $a(p p) \subseteq X$ for every plural propositional variable $p p$.

For any model, define a function $\llbracket \cdot \rrbracket^{\cdot}$ which maps any formula $\varphi$ and assignment function $a$ to some $\llbracket \varphi \rrbracket^{a} \in X$, as follows (where $\llbracket \varphi \rrbracket_{l}^{a}$ and $\llbracket \varphi \rrbracket_{\tau}^{a}$ abbreviate $l\left(\llbracket \varphi \rrbracket^{a}\right.$ ) and $\tau\left(\llbracket \varphi \rrbracket^{a}\right)$, respectively):

$$
\begin{aligned}
& \llbracket p \rrbracket^{a}=a(p) \\
& \llbracket \neg \varphi \rrbracket^{a}=\tilde{c}\left\langle\llbracket \varphi \rrbracket_{l}^{a}, 1-\llbracket \varphi \rrbracket_{\tau}^{a}\right\rangle \\
& \llbracket \varphi \wedge \psi \rrbracket^{a}=\tilde{c}\left\langle\left\{\llbracket \varphi \rrbracket^{a}, \llbracket \psi \rrbracket^{a}: s=o\right\}, \min \left\{\llbracket \varphi \rrbracket_{\tau}^{a}, \llbracket \psi \rrbracket_{\tau}^{a}\right\}\right\rangle \\
& \llbracket \varphi \vee \psi \rrbracket^{a}=\tilde{c}\left\langle\left\{\llbracket \varphi \rrbracket^{a}, \llbracket \psi \rrbracket^{a}: s=o\right\}, \max \left\{\llbracket \varphi \rrbracket_{\tau}^{a}, \llbracket \psi \rrbracket_{\tau}^{a}\right\}\right\rangle \\
& \llbracket \forall p \varphi \rrbracket^{a}=\tilde{c}\left\langle\left\{\llbracket \varphi \rrbracket^{a[x / p]}: x \in X, s=i\right\}, \min \left\{\llbracket \varphi \rrbracket_{\tau}^{a[x / p]}: x \in X\right\}\right\rangle \\
& \llbracket \exists p \varphi \rrbracket^{a}=\tilde{c}\left\langle\left\{\llbracket \varphi \rrbracket^{a[x / p]}: x \in X, s=i\right\}, \max \left\{\llbracket \varphi \rrbracket_{\tau}^{a[x / p]}: x \in X\right\}\right\rangle \\
& \llbracket \forall p p \varphi \rrbracket^{a}=\tilde{c}\left\langle\left\{\llbracket \varphi \rrbracket^{a[Y / p p]}: Y \subseteq X\right\}, \min \left\{\llbracket \varphi \rrbracket_{\tau}^{a[Y / p p]}: Y \subseteq X\right\}\right\rangle \\
& \llbracket \exists p p \varphi \rrbracket^{a}=\tilde{c}\left\langle\left\{\llbracket \varphi \rrbracket^{a[Y / p p]}: Y \subseteq X\right\}, \max \left\{\llbracket \varphi \rrbracket_{\tau}^{a[Y / p p]}: Y \subseteq X\right\}\right\rangle \\
& \llbracket \varphi \prec p p \rrbracket^{a}=\tilde{c}\left\langle\emptyset,\left\{\emptyset: \llbracket \varphi \rrbracket^{a} \in a(p p)\right\}\right\rangle .
\end{aligned}
$$

Define $\mathfrak{M}, a \vDash \varphi$ if $\llbracket \varphi \rrbracket_{\tau}^{a}=1 . \varphi$ is valid in $\mathfrak{M}$, written $\mathfrak{M} \vDash \varphi$, if $\mathfrak{M}, a \vDash \varphi$ for all assignment functions $a$. Define $\vDash \varphi$ if $\mathfrak{M} \vDash \varphi$ for all models $\mathfrak{M}$.

The clauses for negation and quantifiers can usefully be re-stated using the following definitions:

Definition 11. In any model, define for all $x \in X, f: X \rightarrow X$ and $Y \subseteq X$ :

$$
\begin{aligned}
& -x:=c\langle l(x), 1-\tau(x)\rangle \\
& f[Y]:=\{f(x): x \in Y\} \\
& \operatorname{Inst}_{p}^{a}(\varphi):=\left\{\llbracket \varphi \rrbracket^{a[x / p]}: x \in X\right\} \\
& \operatorname{Inst}_{p p}^{a}(\varphi):=\left\{\llbracket \varphi \rrbracket^{a[Y / p p]}: Y \subseteq X\right\} .
\end{aligned}
$$

With this, the above interpretation clauses are equivalent to the following, assuming, in the case of the second, that $s=i$ (and similarly for existential quantifiers): 


$$
\begin{aligned}
& \llbracket \neg \varphi \rrbracket^{a}=-\llbracket \varphi \rrbracket^{a} \\
& \llbracket \forall p \varphi \rrbracket^{a}=\tilde{c}\left\langle\operatorname{Inst}_{p}^{a}(\varphi), \min \tau\left[\operatorname{Inst}_{p}^{a}(\varphi)\right]\right\rangle \\
& \llbracket \forall p p \varphi \rrbracket^{a}=\tilde{c}\left\langle\operatorname{Inst}_{p p}^{a}(\varphi), \min \tau\left[\operatorname{Inst}_{p p}^{a}(\varphi)\right]\right\rangle .
\end{aligned}
$$

To be able to use models in consistency proofs, $\vdash$ first needs to be shown to be sound with respect to validity. This relies on the following standard lemma:

LEMMA 12. In any model: First, $\llbracket \varphi \rrbracket^{a}=\llbracket \varphi \rrbracket^{b}$ whenever a and b agree on the free variables in $\varphi$. Second, for every variable $v$ (propositional or plural propositional), $\llbracket \varphi[\varepsilon / v] \rrbracket^{a}=$ $\llbracket \varphi \rrbracket^{\left.a \llbracket[\varepsilon]^{a} / v\right]}$.

Proof. By inductions on the complexity of $\varphi$.

Furthermore, soundness relies on connectives (primitive and defined) to obey the standard truth-conditions:

LEMMA 13. For any model $\mathfrak{M}$ and assignment function a:

$$
\begin{aligned}
& \mathfrak{M}, a \vDash \neg \varphi \text { iff } \mathfrak{M}, a \not \models \varphi \\
& \mathfrak{M}, a \vDash \varphi \wedge \psi \text { iff } \mathfrak{M}, a \vDash \varphi \text { and } \mathfrak{M}, a \vDash \psi \\
& \mathfrak{M}, a \vDash \varphi \vee \psi \text { iff } \mathfrak{M}, a \vDash \varphi \text { or } \mathfrak{M}, a \vDash \psi \\
& \mathfrak{M}, a \vDash \forall p \varphi \text { iff } \mathfrak{M}, a[x / p] \vDash \varphi \text { for all } x \in X \\
& \mathfrak{M}, a \vDash \exists p \varphi \text { iff } \mathfrak{M}, a[x / p] \vDash \varphi \text { for some } x \in X \\
& \mathfrak{M}, a \vDash \forall p p \varphi \text { iff } \mathfrak{M}, a[Y / p p] \vDash \varphi \text { for all } Y \subseteq X \\
& \mathfrak{M}, a \vDash \exists p p \varphi \text { iff } \mathfrak{M}, a[Y / p p] \vDash \varphi \text { for some } Y \subseteq X \\
& \mathfrak{M}, a \vDash \varphi \prec p p \text { iff } \llbracket \varphi \rrbracket^{a} \in a(p p) \\
& \mathfrak{M}, a \vDash \varphi \rightarrow \psi \text { iff } \mathfrak{M}, a \vDash \varphi \text { only if } \mathfrak{M}, a \vDash \psi \\
& \mathfrak{M}, a \vDash \varphi \leftrightarrow \psi \text { iff } \mathfrak{M}, a \vDash \varphi \text { iff } \mathfrak{M}, a \vDash \psi \\
& \mathfrak{M}, a \vDash \varphi=\psi \text { iff } \llbracket \varphi \rrbracket^{a}=\llbracket \psi \rrbracket^{a} \\
& \mathfrak{M}, a \vDash p p=q q \text { iff } a(p p)=a(q q) \text {. }
\end{aligned}
$$

Proof. By the constraints on models, in general $\tau \tilde{c}\langle I, t\rangle=t$. Thus, if $\llbracket \varphi \rrbracket^{a}=$ $\tilde{c}\langle I, t\rangle$, then $\llbracket \varphi \rrbracket_{\tau}^{a}=t$. With this, the claims for the primitive connectives follow straightforwardly from the definition of $\llbracket \cdot \rrbracket$. By way of example, consider the case of negation:

$$
\mathfrak{M}, a \vDash \neg \varphi \text { iff } \llbracket \neg \varphi \rrbracket_{\tau}^{a}=1 \text { iff } 1-\llbracket \varphi \rrbracket_{\tau}^{a}=1 \text { iff } \llbracket \varphi \rrbracket_{\tau}^{a} \neq 1 \text { iff } \mathfrak{M}, a \not \models \varphi .
$$

The cases of the defined connectives follow from their definitions and the cases of the primitive connectives as usual.

Proposition 14 (Soundness). If $\vdash \varphi$, then $\vDash \varphi$.

Proof. By induction on the length of proofs, using the previous two lemmas.

It remains to identify models which validate $T_{o}$ and $T_{i}$. $\mathrm{O}_{\neg}$ can in fact be shown to be valid in all models, as - is guaranteed to be an injection:

Lemma 15. In any model, - is injective.

Proof. If $-x=-y$, then

$$
c\left\langle\pi_{1} c^{-1}(x), 1-\pi_{2} c^{-1}(x)\right\rangle=c\left\langle\pi_{1} c^{-1}(y), 1-\pi_{2} c^{-1}(y)\right\rangle .
$$

Since $c$ is bijective, it follows that $\pi_{1} c^{-1}(x)=\pi_{1} c^{-1}(y)$ and $1-\pi_{2} c^{-1}(x)=1-\pi_{2} c^{-1}(y)$. Thus $c^{-1}(x)=c^{-1}(y)$, whence $x=y$. 
Proposition 16. $\vDash \mathrm{O}_{\neg}$.

Proof. Since $\llbracket \neg \varphi \rrbracket^{a}=-\llbracket \varphi \rrbracket^{a}$, the claim follows from Lemma 15.

5.2. Operand models. This section shows $T_{o}$ to be consistent. First, it will be shown that any model in which the switch $s$ is $o$ validates $\mathrm{I}_{Q p p}$, for universal and existential plural propositional quantifiers, and $\mathrm{O}_{\circ}^{-}$for conjunction and disjunction. Using a syntactic mapping, this is then extended to $\mathrm{O}_{\circ}$.

Definition 17. Let an operand model be a model $\langle X, W, c, s\rangle$ such that $s=o$.

LeMma 18. There exist operand models.

Proof. If $X$ is an infinite set and $W$ the set of finite subsets of $X$, then for cardinality reasons, there exists a bijection $c$ from $W \times\{0,1\}$ to $X$, and so an operand model $\langle X, W, c, o\rangle$.

Starting with $\mathrm{I}_{Q p p}$, the crucial step is to show that $\operatorname{Inst}_{p p}^{a}(\varphi)$ is always well-behaved. This is best shown by proving that for every formula $\varphi$ and assignment function $a$, the set of propositions $\llbracket \varphi \rrbracket^{b}$ is finite, where $b$ may be any assignment function differing from $a$ in the interpretation of plural propositional variables.

DeFINITION 19. In any model, define:

$$
\operatorname{Inst}_{\pi}^{a}(\varphi):=\left\{\llbracket \varphi \rrbracket^{a[\bar{Y} / \bar{p} p]}: \bar{Y} \subseteq X\right\},
$$

where $\overline{p p}$ abbreviates $p p_{1}, \ldots, p p_{n}$ (the sequence of free plural propositional variables in $\varphi$ ), $\bar{Y}$ abbreviates $Y_{1}, \ldots, Y_{n}$, and $\bar{Y} \subseteq X$ abbreviates $Y_{1} \subseteq X, \ldots, Y_{n} \subseteq X$.

LEMMA 20. In any operand model, for every formula $\varphi$ and every assignment function a, $\operatorname{Inst}_{\pi}^{a}(\varphi)$ is finite.

Proof. By induction on the complexity of $\varphi$. Exemplarily, consider two cases:

Assume $\varphi$ is a conjunction $\psi \wedge \chi$. $\operatorname{Inst}_{\pi}^{a}(\psi \wedge \chi)$ is a subset of:

$$
\left\{\tilde{c}\left\langle\left\{\llbracket \psi \rrbracket^{a[\bar{Y} / \bar{p} p]}, \llbracket \chi \rrbracket^{a[\bar{Y} / \bar{p} p]}\right\}, t\right\rangle: \bar{Y} \subseteq X, t<2\right\} .
$$

This, in turn, is a subset of $\left\{\tilde{c}\langle\{x, y\}, t\rangle: x, y \in \operatorname{Inst}_{\pi}^{a}(\psi) \cup \operatorname{Inst}_{\pi}^{a}(\chi), t<2\right\}$, which by IH is finite.

Assume $\varphi$ is a universal plural propositional quantification $\forall q q \psi . \operatorname{Inst}_{\pi}^{a}(\forall q q \psi)$ is a subset of:

$$
\left\{\tilde{c}\left\langle\left\{\llbracket \psi \rrbracket^{a[\bar{Y} / \bar{p} p][Z / q q]}: Z \subseteq X\right\}, t\right\rangle: \bar{Y} \subseteq X, t<2\right\} .
$$

This, in turn, is a subset of $\left\{\tilde{c}\langle I, t\rangle: I \subseteq \operatorname{Inst}_{\pi}^{a}(\psi), t<2\right\}$, which by IH is finite.

With this, the bijectivity of $c$ ensures the validity of $\mathrm{I}_{Q p p}$, for plural propositional quantifiers:

Proposition 21. For each plural propositional quantifier $Q$, $\mathrm{I}_{Q p p}$ is valid in every operand model.

Proof. Consider the universal case; the existential case is analogous.

If $\mathfrak{M}, a \vDash \forall p p \varphi(p p)=\forall p p \psi(p p)$, then for some $t_{1}, t_{2}<2$ :

$$
\tilde{c}\left\langle\operatorname{Inst}_{p p}^{a}(\varphi(p p)), t_{1}\right\rangle=\tilde{c}\left\langle\operatorname{Inst}_{p p}^{a}(\psi(p p)), t_{2}\right\rangle .
$$


By Lemma 20, $\operatorname{Inst}_{p p}^{a}(\varphi(p p))$ and $\operatorname{Inst}_{p p}^{a}(\psi(p p))$ are finite, and so members of $W$. Thus by the injectivity of $c$, Inst ${ }_{p p}^{a}(\varphi(p p))=$ Inst $_{p p}^{a}(\psi(p p))$. So for every $Y \subseteq X$ there is a $Z \subseteq$ $X$ such that $\llbracket \varphi(p p) \rrbracket^{a[Y / p p]}=\llbracket \psi(p p) \rrbracket^{a[Z / p p]}$. By Lemma 12, the latter is $\llbracket \psi(q q) \rrbracket^{a[Z / q q]}$. Thus $\mathfrak{M}, a \vDash \forall p p \exists q q(\varphi(p p)=\psi(q q))$.

A similar argument shows the validity of $O_{\wedge}^{-}$and $O_{\vee}^{-}$, as $\left\{\llbracket \varphi \rrbracket^{a}, \llbracket \psi \rrbracket^{a}\right\}$ must be finite and so well-behaved:

Proposition 22. $O_{\wedge}^{-}$and $O_{\vee}^{-}$are valid in every operand model.

Proof. Consider the conjunctive case; the disjunctive case is analogous.

Assume $\mathfrak{M}, a \vDash p \wedge p^{\prime}=q \wedge q^{\prime}$. Then for some $t_{1}, t_{2}<2$ :

$$
\tilde{c}\left\langle\left\{a(p), a\left(p^{\prime}\right)\right\}, t_{1}\right\rangle=\tilde{c}\left\langle\left\{a(q), a\left(q^{\prime}\right)\right\}, t_{2}\right\rangle .
$$

As $\left\{a(p), a\left(p^{\prime}\right)\right\}$ and $\left\{a(q), a\left(q^{\prime}\right)\right\}$ are finite, they are members of $W$. It therefore follows from the bijectivity of $c$ that $\left\{a(p), a\left(p^{\prime}\right)\right\}=\left\{a(q), a\left(q^{\prime}\right)\right\}$. By elementary set theory, it follows that either $a(p)=a(q)$ and $a\left(p^{\prime}\right)=a\left(q^{\prime}\right)$, or $a(p)=a\left(q^{\prime}\right)$ and $a\left(p^{\prime}\right)=a(q)$. Thus $\mathfrak{M}, a \vDash\left(p=q \wedge p^{\prime}=q^{\prime}\right) \vee\left(p=q^{\prime} \wedge p^{\prime}=q\right)$.

By soundness, operand models therefore witness the consistency of $\left\{\mathrm{O}_{\neg}, \mathrm{O}_{\wedge}^{-}, \mathrm{O}_{\vee}^{-}\right.$, $\left.\mathrm{I}_{\forall p p}, \mathrm{I}_{\exists p p}\right\}$ in $\vdash$. In order to extend this consistency result to $T_{o}$, recall how any binary connective $\circ$ satisfying $\mathrm{O}_{\circ}^{-}$can be used to define a binary connective ô satisfying $\mathrm{O}_{\hat{\circ}}$. $\circ$ and ô are not truth-functionally equivalent, but given both $\mathrm{O}_{\wedge}^{-}$and $\mathrm{O}_{\vee}^{-}$, a variant definition ${ }^{-}$is available which is truth-functionally equivalent to $\circ$, with $\mathrm{O}_{\circ}^{-}$still entailing $\mathrm{O}_{\bar{\circ}}$. This can be used to define a function mapping any formula $\varphi$ to a formula $\bar{\varphi}$ which replaces any occurrence of $\wedge$ and $\vee$ by $\bar{\wedge}$ and $\bar{V}$, respectively. It can be shown that if $\varphi$ is entailed by $T_{o}$, then $\bar{\varphi}$ is valid in any operand model, and this suffices for consistency.

o can be defined as follows:

$$
\varphi \bar{\circ} \psi:=((\varphi \wedge \neg \varphi) \vee(\varphi \wedge \varphi)) \circ(\psi \wedge \psi) .
$$

By TAUT, for $\circ \in\{\wedge, \vee\}, \vdash \varphi \bar{\circ} \psi \leftrightarrow \varphi \circ \psi$. Define $\bar{\varphi}$ recursively, so that ' maps every atomic formulas to itself, commutes with all logical constants except $\wedge$ and $\vee$, and satisfies the following two clauses:

$$
\begin{aligned}
& \overline{\varphi \wedge \psi}:=\bar{\varphi} \bar{\wedge} \bar{\psi} \\
& \overline{\varphi \vee \psi}:=\bar{\varphi} \bar{\nabla} \overline{.}
\end{aligned}
$$

To show that this has the intended effect, it suffices to show that if $T_{o} \vdash \varphi$, then $\bar{\varphi}$ is valid in any operand model. With the results above, this follows from the following three lemmas:

LeMma 23. For any quantifier $Q v, \mathrm{I}_{Q v} \vdash \overline{\mathrm{I}_{Q v}}$, and $\mathrm{O}_{\neg} \vdash \overline{\mathrm{O}_{\neg}}$.

Proof. Consider exemplarily the case of a quantifier $Q v$; the remaining case of negation is analogous.

Let $\varphi$ be an instance of $\mathrm{I}_{Q v}$ for complement clauses $\psi$ and $\chi$. Then $\bar{\varphi}$ is provably equivalent to

$$
Q v \bar{\psi}(v)=Q v \bar{\chi}(v) \rightarrow \forall v \exists v^{\prime}\left(\bar{\psi}(v)=\bar{\chi}\left(v^{\prime}\right)\right),
$$

which is an instance of $\mathrm{I}_{Q v}$.

LEMMA 24. $\mathrm{O}_{\wedge}^{-} \wedge \mathrm{O}_{\vee}^{-} \vdash \overline{\mathrm{O}_{\wedge}} \wedge \overline{\mathrm{O}_{\vee}}$. 
Proof. Analogous to Lemma $6, \mathrm{O}_{\wedge}^{-} \wedge \mathrm{O}_{\vee}^{-} \vdash \mathrm{O}_{\bar{\wedge}} \wedge \mathrm{O}_{\bar{\nabla}}$. With this, the claim follows from the fact that $\mathrm{O}_{\bar{\wedge}} \wedge \mathrm{O}_{\bar{\vee}} \vdash \overline{\mathrm{O}_{\wedge}} \wedge \overline{\mathrm{O}_{\vee}}$.

LEMMA 25. If $\vdash \varphi$ then $\vdash \bar{\varphi}$.

Proof. By induction on the length of proofs. For every instance $\varphi$ of an axiom schema of $\vdash$, there is an instance $\varphi^{\prime}$ which is equivalent to $\bar{\varphi}$. For example,

$$
\vdash \overline{(\forall v \psi \rightarrow \psi[\varepsilon / v])} \leftrightarrow(\forall v \bar{\psi} \rightarrow \bar{\psi}[\bar{\varepsilon} / v]) .
$$

Analogously for the two rules of $\vdash$. For example, assume that $\vdash \psi$ using MP from $\vdash \varphi$ and $\vdash \varphi \rightarrow \psi$. By IH,$\vdash \bar{\varphi}$ and $\vdash \overline{\varphi \rightarrow \psi}$. By the latter, $\vdash \bar{\varphi} \rightarrow \bar{\psi}$, so using $\mathrm{MP}, \vdash \bar{\psi}$.

THEOREM 26. $T_{o}$ is consistent.

Proof. By Lemma 18, there exists an operand model $\mathfrak{M}$. As $p$ is $\bar{p}, \mathfrak{M} \not \models \bar{p}$. So it suffices to show that $T_{o} \vdash \varphi$ only if $\mathfrak{M} \vDash \bar{\varphi}$. And this follows from previous results (using soundness throughout): By Propositions 16 and 21 and Lemma 23, $\mathfrak{M} \vDash \bar{\varphi}$ whenever $\varphi$ is $\mathrm{O}_{\neg}$ or $\mathrm{I}_{Q p}$. By Proposition 22 and Lemma 24, $\mathfrak{M} \vDash \bar{\varphi}$ whenever $\varphi$ is $\mathrm{O}_{\wedge}$ or $\mathrm{O}_{\vee}$. So $\varphi \in T_{o}$ only if $\mathfrak{M} \vDash \bar{\varphi}$. That $T_{o} \vdash \varphi$ only if $\mathfrak{M} \vDash \bar{\varphi}$ follows with Lemma 25.

5.3. Instance models. To show that $T_{i}$ is consistent, models will be used in which the switch $s$ is set to $i$. This ensures that the interpretational clauses of quantified formulas behave as expected, with $\llbracket \forall p \varphi \rrbracket^{a}$ being interpreted as a proposition determined by Inst $_{p}^{a}(\varphi)$ and the minimum of the truth-values of these instances. However, for $\mathrm{I}_{Q v}$ to be valid, for all quantifiers, it must be shown that $\operatorname{Inst}_{v}^{a}(\varphi)$ is always well-behaved. And this requires $W$ to contain some infinite sets.

To illustrate this, consider the formula $\forall p p$. $\operatorname{Inst}_{p}^{a}(p)$ is simply $X$, the set of all propositions, so $X$ must be well-behaved. Similarly, consider $\forall p \forall q p$. For every $p$, the proposition expressed by $\forall q p$ is a distinct proposition with a single instance $p$, so Inst ${ }_{p}^{a}(\forall q p)$ is the infinite set of these propositions, which must be well-behaved as well. Similarly, $\operatorname{Inst}_{p}^{a}(\neg \forall q p)$, the set of instances of the proposition expressed by $\forall p \neg \forall q p$, is the set of negations of these propositions; this must also be well-behaved. Since quantifiers and negations can be nested, $W$ must more generally be required to be closed under correspondingly iterated operations on sets of propositions, in addition to containing $X$. The next definition formulates these constraints in suitable generality. To state it, for any set $A, A^{*}$ is taken to be the set of finite strings of elements of $A$, i.e., $\bigcup_{n<\omega} A^{n}$, and $e$ the string of elements of length 0 .

Definition 27. Let an instance model be a model $\langle X, W, c, s\rangle$ such that $s=i$ and $c^{\sigma}[X] \in W$ for all $\sigma \in\{q, n\}^{*}$, where, for all $x \in X$ :

$$
\begin{aligned}
& c^{e}(x)=x, \\
& c^{\sigma q}(x)=c\left\langle\left\{c^{\sigma}(x)\right\}, \tau c^{\sigma}(x)\right\rangle, \\
& c^{\sigma n}(x)=-c^{\sigma}(x) .
\end{aligned}
$$

Cardinality considerations again suggest that these constraints are satisfiable. Starting from a countably infinite set $X$, the set of finite subsets of $X$ is countable. And as $\{q, n\}^{*}$ is countable, so is the set $\left\{c^{\sigma}[X]: \sigma \in\{q, n\}^{*}\right\}$, given any choice of $c$. Thus, $W$ can be chosen to be countable, so that there exists a bijection $c$ from $W \times\{0,1\}$ to $X$. The only difficulty is that in this line of reasoning, the choice of $W$ is dependent on a choice of $c$, which itself depends on $W$. The difficulty can be overcome 
by dividing $X$ into four countably infinite subsets: the set $X_{f}^{1}$ of true propositions with finitely many instances, the set $X_{\infty}^{1}$ of true propositions with infinitely many instances, and correspondingly sets $X_{f}^{0}$ and $X_{\infty}^{0}$ of false propositions. Fixing the interpretation of negation by a suitable function $\sim$ on $X$, the choice of $c$ and $W$ can be determined in three steps: First, the behavior of $c$ can be fixed for pairs $\langle I, t\rangle$ with $I$ finite, by choosing a suitable bijection with codomain $X_{f}^{1} \cup X_{f}^{0}$. Second, $W$ is determined by this, since $c^{\sigma}$ is determined by the behavior of $c$ on such pairs and the behavior of negation. Finally, $c$ can be completed by choosing a suitable bijection from pairs $\langle I, t\rangle$ with $I$ an infinite member of $W$ to $X_{\infty}^{1} \cup X_{\infty}^{0}$. The following proof makes this line of argument precise.

LEMMA 28. There exist instance models.

Proof. Let $X$ be a countably infinite set. Partition $X$ into four infinite subsets $X_{f}^{0}$, $X_{f}^{1}, X_{\infty}^{0}$ and $X_{\infty}^{1}$. By omitting one parameter of these terms, the union of the two choices is indicated; e.g., $X_{\infty}=X_{\infty}^{0} \cup X_{\infty}^{1}$. Let $\sim$ be an involution on $X$ such that $\sim \mid X_{f}^{0}$ is a bijection with codomain $X_{f}^{1}$, and $\sim \mid X_{\infty}^{0}$ is a bijection with codomain $X_{\infty}^{1}$.

Let $W_{f}$ be the set of finite subsets of $X$, and let $c_{f}: W_{f} \times\{0,1\} \rightarrow X_{f}$ such that $c_{f} \mid W_{f} \times\{0\}$ is a bijection with codomain $X_{f}^{0}$, and for all $I \in W_{f}, c_{f}\langle I, 1\rangle=\sim c_{f}\langle I, 0\rangle$. For each $\sigma \in\{q, n\}^{*}$, define $g^{\sigma}: X \rightarrow X$ such that:

$$
\begin{aligned}
& g^{e}(x)=x, \\
& g^{\sigma q}(x)=c_{f}\left\langle\left\{g^{\sigma}(x)\right\},\left\{\emptyset: g^{\sigma}(x) \in X^{1}\right\}\right\rangle, \\
& g^{\sigma n}(x)=\sim g^{\sigma}(x) .
\end{aligned}
$$

Define $W_{\infty}=\left\{g^{\sigma}[X]: \sigma \in\{q, n\}^{*}\right\}$. It can be shown that $W_{\infty}$ is countably infinite and disjoint from $W_{f}$ : To show that $W_{\infty}$ is infinite, one shows by an induction on the length of sequences that for each $\sigma \in\{q\}^{*}, g^{\sigma q}[X] \subsetneq g^{\sigma}[X]$. The inclusion is immediate. That the inclusion is proper is straightforward in the base case. For the induction step, by IH, there is some $x \in g^{\sigma}[X]$ such that $x \notin g^{\sigma q}[X]$. Then $g^{q}(x) \in g^{\sigma q}[X]$, and as $c_{f}$ is a bijection, $g^{q}(x) \notin g^{\sigma q q}[X]$. $W_{\infty}$ is countable by construction. To prove that $W_{\infty}$ is disjoint from $W_{f}$, it suffices to shown that for each $\sigma \in\{q, n\}^{*}, g^{\sigma}[X] \notin W_{f}$. This can be done by another induction on the length of sequences appealing to the bijectivity of $c_{f}$.

Let $c_{\infty}: W_{\infty} \times\{0,1\} \rightarrow X_{\infty}$ such that $c_{\infty} \mid W_{\infty} \times\{0\}$ is a bijection with codomain $X_{\infty}^{0}$, and for all $Y \in W_{\infty}, c_{\infty}\langle Y, 1\rangle=\sim c_{\infty}\langle Y, 0\rangle$. Let $W=W_{f} \cup W_{\infty}$ and $c=c_{f} \cup$ $c_{\infty}$. It remains to show that $\mathfrak{M}=\langle X, W, c, i\rangle$ is an instance model.

Since $c_{f}$ is a bijection from $W_{f} \times\{0,1\}$ to $X_{f}$, and $c_{\infty}$ is a bijection from $W_{\infty} \times$ $\{0,1\}$ to $X_{\infty}, c$ is a bijection from $W \times\{0,1\}$ to $X$. Thus, $\mathfrak{M}$ is a model $\langle X, W, c, s\rangle$ with $s=i$, and so it suffices to show that $c^{\sigma}[X] \in W$, for all $\sigma \in\{q, n\}^{*}$. This, in turn, follows from the claim that $g^{\sigma}=c^{\sigma}$ for all $\sigma \in\{q, n\}^{*}$, which is established by induction on the length of $\sigma$ :

(e) Immediate.

$(\sigma q)$ Consider any $x \in X$. By construction and $\mathrm{IH}, g^{\sigma q}(x)$ is

$$
c\left\langle\left\{c^{\sigma}(x)\right\},\left\{\emptyset: c^{\sigma}(x) \in X^{1}\right\}\right\rangle .
$$

For any $y \in X, y \in X^{1}$ iff $\tau(y)=1$. Thus $\left\{\emptyset: c^{\sigma}(x) \in X^{1}\right\}=\tau c^{\sigma}(x)$. It follows that $g^{\sigma q}(x)=c^{\sigma q}(x)$, as required.

$(\sigma n)$ It suffices to show, for any $x \in X$, that $\sim x=-x$. Recall that $c_{f}$ and $c_{\infty}$ were chosen so as to guarantee that $c(I, 1)=\sim c(I, 0)$ for every $I \in W$. Since 
$\sim$ is an involution, it follows that also $c(I, 0)=\sim c(I, 1)$. So, for any $x \in X$, $\sim x=\sim c\langle l(x), \tau(x)\rangle=c\langle l(x), 1-\tau(x)\rangle=-x$.

Having defined instance models and demonstrated their existence, the next step is to show that they behave as intended, i.e., that $\operatorname{Inst}_{v}^{a}(\varphi)$ is always well-behaved. This follows from the following lemma:

LEMMA 29. For every formula $\varphi$, either

(i) there is a finite set $F \subseteq X$ such that for all assignment functions $a, \llbracket \varphi \rrbracket^{a} \in F$, or

(ii) there is a string $\sigma \in\{q, n\}^{*}$ and propositional variable $p$ such that for all assignment functions $a, \llbracket \varphi \rrbracket^{a}=c^{\sigma} a(p)$.

Proof. By induction on the complexity of $\varphi$.

( $p$ ) If $\varphi$ is a variable $p$, then $\llbracket p \rrbracket^{a}=a(p)=c^{e} a(p)$, so $e$ and $p$ witness case (ii).

$(\neg \psi)$ Assume $\varphi$ is $\neg \psi$. By IH, one of cases (i) and (ii) obtains for $\psi$.

Case (i): There is a finite set $F \subseteq X$ such that for all assignment functions $a, \llbracket \psi \rrbracket^{a} \in F$. Then $-[F]$ is finite and contains $\llbracket \neg \psi \rrbracket^{a}$, for all assignment functions $a$.

Case (ii): There is a string $\sigma \in\{q, n\}^{*}$ and variable $p$ such that for all assignment functions $a, \llbracket \psi \rrbracket^{a}=c^{\sigma} a(p)$. So for all assignment functions $a$, $\llbracket \neg \psi \rrbracket^{a}=-c^{\sigma} a(p)=c^{\sigma n} a(p)$. So $\sigma n$ and $p$ witness case (ii).

$(\wedge, \vee, \prec)$ If $\varphi$ is of the form $\psi \wedge \chi, \psi \vee \chi$ or $\psi \prec p p$, then for any assignment function $a, \llbracket \varphi \rrbracket^{a} \in\{c\langle\emptyset, 0\rangle, c\langle\emptyset, 1\rangle\}$, which is finite.

$(\forall v \psi)$ For any assignment function $a, \mathbb{\llbracket} \forall v \psi \rrbracket^{a}=c\left\langle\operatorname{Inst}_{v}^{a}(\psi), \min \tau\left[\operatorname{Inst}_{v}^{a}(\psi)\right]\right\rangle$. By induction hypothesis, one of cases (i) and (ii) obtains for $\psi$.

Case (i): There is a finite set $F \subseteq X$ such that for all assignment functions $a, \llbracket \psi \rrbracket^{a} \in F$. So $\operatorname{Inst}_{v}^{a}(\psi) \subseteq F$; it follows that for every assignment function $a$, $\llbracket \forall v \psi \rrbracket^{a}$ is a member of the finite set $F^{\prime}=\{c\langle I, t\rangle: I \subseteq F, t<2\}$.

Case (ii): There is a string $\sigma \in\{q, n\}^{*}$ and variable $p$ such that for all assignment functions $a, \llbracket \psi \rrbracket^{a}=c^{\sigma} a(p)$. Distinguish two sub-cases: If $v=$ $p$, then $\operatorname{Inst}_{v}^{a}(\psi)=c^{\sigma}[X]$, whence $\llbracket \forall v \psi \rrbracket^{a}=\tilde{c}\left\langle c^{\sigma}[X], \min \tau\left[c^{\sigma}[X]\right]\right\rangle$. So the singleton of this element witnesses case (i). If $v \neq p$, then $\operatorname{Inst}_{v}^{a}(\psi)=\left\{c^{\sigma} a(p)\right\}$, whence $\llbracket \forall v \psi \rrbracket^{a}=\tilde{c}\left\langle\left\{c^{\sigma} a(p)\right\}, \tau c^{\sigma} a(p)\right\rangle=c^{\sigma q} a(p)$. So $\sigma q$ and $p$ witness case (ii).

$(\exists v \psi)$ Analogous to the universal case.

LEMma 30. In any instance model, for every assignment function a, variable $v$ (propositional or plural propositional) and formula $\varphi, \operatorname{Inst}_{v}^{a}(\varphi) \in W$.

Proof. Consider any formula $\varphi$. Using Lemma 29, distinguish two cases:

Case (i): There is a finite set $F \subseteq X$ such that for all assignment functions $a$, $\llbracket \varphi \rrbracket^{a} \in$ $F$. So $\operatorname{Inst}_{v}^{a}(\varphi)$ is a subset of $F$, and so finite, and therefore a member of $W$.

Case (ii): There is a string $\sigma \in\{q, n\}^{*}$ and propositional variable $p$ such that for all assignment functions $a, \llbracket \varphi \rrbracket^{a}=c^{\sigma} a(p)$. Distinguish two sub-cases: If $v=p$, then it follows from $\llbracket \varphi \rrbracket^{a}=c^{\sigma} a(p)$ that $\operatorname{Inst}_{v}^{a}(\varphi)=c^{\sigma}[X]$, which is a member of $W$. If $v \neq p$, then $\operatorname{Inst}_{v}^{a}(\varphi)=\left\{c^{\sigma} a(p)\right\}$, which is finite, and so a member of $W$ as well.

Proposition 31. For each quantifier $Q v, \mathrm{I}_{Q v}$ is valid in every instance model.

Proof. Analogous to the proof of Proposition 21, using Lemma 30. 
THEOREM 32. $T_{i}$ is consistent.

Proof. By Lemma 28, there is an instance model $\mathfrak{M}$. By Propositions 16 and 31, all members of $T_{i}$ are valid in $\mathfrak{M}$. The claim follows by soundness (Proposition 14).

§6. Refinements. The results established here show that the consistency of logical structure is a somewhat subtle matter: while partial theories like $T_{o}$ and $T_{i}$ are consistent, already the inclusion of $\mathrm{O}_{\circ}^{-}$and $\mathrm{I}_{Q p}$ for a binary operator $\circ$ and a propositional quantifier $Q p$ leads to inconsistency. The consistency of $T_{o}$ and $T_{i}$ is of some technical interest, but philosophically, the more important finding is presumably the (much simpler) inconsistency of $\mathrm{O}_{\circ}^{-}$and $\mathrm{I}_{Q p}$ for a binary operator $\circ$ and a propositional quantifier $Q p$ : those who think that propositions exhibit logical structure will presumably hold that this applies to sentential operators and quantifiers alike. Thus, it is natural to consider ways of avoiding inconsistency while at the same time upholding some form of logical structure for both sentential operators and quantifiers. This section considers two avenues in this direction; the first considers weakening the background logic, and the second weakening the logical structure principles.

6.1. Weakening logic. The axiomatic principles of $\vdash$ comprise, apart from elementary principles governing Boolean connectives and quantifiers, two principles which are specific to plural quantification: PC and ExT. Weakening the elementary principles governing Boolean connectives and quantifiers won't be considered here; many will consider them far more plausible than any principle of logical structure. The following therefore considers dropping or weakening one of ExT and PC.

Inspecting the deduction of an inconsistency sketched in Section 4, it is easy to see that ExT is nowhere appealed to. There is thus nothing to be gained by questioning EXT. Furthermore, this shows that this proof could as well have been carried out in a more standard higher-order language in which plural propositional quantification is replaced with quantifiers binding variables taking the syntactic position of sentential operators. However, the models constructed in Section 5 do little to assure us of the viability of $T_{o}$ and $T_{i}$ in such a context: They may show that these theories are consistent in a proof system corresponding to $\vdash$, and so a fortiori consistent in a proof system omitting the axiom corresponding to ExT. But these models essentially validate EXT, which is at least controversial in the case of quantification into operator position: roughly, such quantifiers can be read as ranging over properties of propositions, which are plausibly individuated non-extensionally. This raises the general question of which sets of logical structure principles are consistent in higher-order systems with nonextensional higher-order quantification, including more comprehensive type theories in which any finite sequence of types gives rise to a type of relational terms.

Consider now the option of restricting PC. The option of restricting PC to PC' was already noted not to restore consistency. Another option is to restrict PC to predicative instances, in which $\varphi$ may not contain any plural propositional quantifiers or parameters. Walsh [15] shows, in a similar type-theoretic setting, that the RussellMyhill theorem essentially relies on impredicative instances of comprehension. This suggests that such a weakening of PC may be enough to render consistent all of the principles of logical structure discussed in Section 3. Assessing this is beyond the scope of this paper, but it is worth noting that even on such a restriction of PC, there are natural variants of $\mathrm{O}_{\circ}^{-}$and $\mathrm{I}_{Q p}$ in an expanded language which are inconsistent. 
To motivate this expansion of the language, note that principles $\mathrm{O}_{\circ}^{-}$and $\mathrm{I}_{Q v}$ allow one to recover operands and instances, in the sense that propositions expressed by applications of $\circ$ are only identical if the operands are the same, and propositions expressed by applications of $Q v$ are only identical if the instances are the same. Those who find such principles attractive may naturally also want to be able to talk of the operands and the instances of any given proposition. Formally, they may thus want to add to the language binary sentential operators $O$ and $I$ satisfying the following principles:

$$
\begin{aligned}
& \left(\mathrm{O}_{\circ}^{O}\right) O(r, p \circ q) \leftrightarrow r=p \vee r=q \\
& \left(\mathrm{I}_{Q v}^{I}\right) I(q, Q v \varphi(v)) \leftrightarrow \exists v(q=\varphi(v)) .
\end{aligned}
$$

In the case of binary operations $\circ$, there is little difference between $\mathrm{O}_{\circ}^{-}$and $\mathrm{O}_{\circ}^{O}$. After all, $\mathrm{O}_{\circ}^{O}$ entails $\mathrm{O}_{\circ}^{-}$, as is easily seen. And conversely, given $\mathrm{O}_{\circ}^{-}$, one can define an operation $O^{\prime}$ satisfying $\mathrm{O}_{\circ}^{O^{\prime}}$, as follows:

$$
O^{\prime}(p, q):=\exists r((p \wedge r)=q \vee(r \wedge p)=q) .
$$

The case of $\mathrm{I}_{Q v}^{I}$ is different. While $\mathrm{I}_{Q v}^{I}$ is still easily seen to entail $\mathrm{I}_{Q v}$, it is not clear how one would even define, on the assumption of $\mathrm{I}_{Q v}$, what it takes for $p$ to be an instance of $q$.

This additional strength of $\mathrm{I}_{Q v}^{I}$ can be harnessed to show an inconsistency result which requires only predicative instances of PC. Instead of the Russell-Myhill theorem, the relevant derivation makes use of the following result, adapted from [14]:

Proposition 33. $\psi(p, \varphi(p p)) \leftrightarrow p \prec p p$ can be shown to be inconsistent using one instance of plural comprehension, namely the one for condition $\neg \psi(q, q)$.

Proof. Assume for contradiction that $\psi(p, \varphi(p p)) \leftrightarrow p \prec p p$. By plural comprehension, there are some $r r$ such that $q \prec r r$ iff $\neg \psi(q, q)$. Then in particular $\varphi(r r) \prec r r$ iff $\neg \psi(\varphi(r r), \varphi(r r))$. But by assumption, $\varphi(r r) \prec r r$ iff $\psi(\varphi(r r), \varphi(r r))$. Contradiction.

Like the Russell-Myhill theorem, this relies on a version of plural comprehension which entails the existence of an empty plurality. But again, this is not essential: if there is no $q$ such that $\neg \psi(q, q)$, then it follows with $\psi(p, \varphi(p p)) \leftrightarrow p \prec p p$ that $\varphi(p p) \nprec p p$ for all $p p$. But this is inconsistent with the existence of the (non-empty) plurality of all propositions, which follows by an instance of predicative comprehension.

Let $\vdash^{-}$be $\vdash$, with PC restricted to predicative instances. In this system, an instance of the schema just shown to be inconsistent can be derived from $\mathrm{O}_{\circ}^{-}$and $\mathrm{I}_{Q p}^{I}$ :

LeMma 34. $\mathrm{O}_{\circ}^{-}, \mathrm{I}_{Q p}^{I} \vdash^{-} \exists q(q \wedge I(q \hat{\circ} p, \forall r(r \prec p p \hat{\circ} r))) \leftrightarrow p \prec p p$.

Proof. Assume $\mathrm{O}_{\circ}^{-}, \mathrm{I}_{Q p}^{I}$. Then $\exists q(q \wedge I(q \hat{\circ} p, \forall r(r \prec p p$ o $r)))$ is equivalent to:

$$
\exists q(q \wedge \exists s((q \hat{\circ} p)=(s \prec p p \hat{o} s))) .
$$

Since the proof of Lemma 6 does not appeal to plural comprehension, this is equivalent to:

$$
\exists q(q \wedge \exists s(q=(s \prec p p) \wedge p=s)) .
$$

This in turn is equivalent to $\exists q(q \wedge q=(p \prec p p))$, which, finally, is equivalent to $p \prec p p$. 
Proposition 35. $\mathrm{O}_{\circ}^{-}$and $\mathrm{I}_{Q p}^{I}$ are jointly predicatively inconsistent.

Proof. By Lemma 34, $\mathrm{O}_{\circ}^{-}$and $\mathrm{I}_{Q p}^{I}$ predicatively entail:

$$
\exists q(q \wedge I(q \hat{\circ} p, \forall r(r \prec p p \hat{\circ} r))) \leftrightarrow p \prec p p .
$$

This is of the form $\psi(p, \varphi(p p)) \leftrightarrow p \prec p p$, for $\psi\left(p, p^{\prime}\right)$ being $\exists q\left(q \wedge I\left(q \hat{\circ} p, p^{\prime}\right)\right)$ and $\varphi(p p)$ being $\forall r(r \prec p p \hat{o} r)$. Since $\exists q\left(q \wedge I\left(q \hat{\circ} p, p^{\prime}\right)\right)$ involves no plural propositional quantifiers or plural propositional parameters, it follows with Proposition 33 that $\mathrm{O}_{\circ}^{-}$ and $\mathrm{I}_{Q p}^{I}$ are jointly inconsistent in $\vdash^{-}$.

While $\mathrm{I}_{Q p}^{I}$ may be stronger than $\mathrm{I}_{Q p}$, it isn't inconsistent on its own, even in $\vdash$, as can be shown by adapting the above model constructions. Interpreting $O$ and $I$ as follows, it is easily seen that operator models and instance models validate $\mathrm{O}_{\circ}^{O}$ and $\mathrm{I}_{Q p}^{I}$ respectively, for binary Boolean connectives $\circ$ and propositional quantifiers $Q p$ :

$$
\llbracket O(\varphi, \psi) \rrbracket^{a}=\llbracket I(\varphi, \psi) \rrbracket^{a}=\tilde{c}\left\langle\emptyset,\left\{\emptyset: \llbracket \varphi \rrbracket^{a} \in \llbracket \psi \rrbracket_{l}^{a}\right\}\right\rangle .
$$

6.2. Weakening logical structure. Consider now the option of weakening the logical structure principles, in order to obtain a consistent theory which nevertheless imposes a natural form of logical structure on propositions. In the case of $\prec$, it is hard to see how any principle weaker than $\mathrm{O}_{\prec}$ would encode the idea that propositions inherit the logical structure of sentences of the form $\varphi \prec p p$. It seems therefore that the idea of logical structure has to be restricted to cases other than those arising from sentences of the form $\varphi \prec p p$. The matter is different in the case of the jointly inconsistent principles $\mathrm{O}_{\circ}^{-}$and $\mathrm{I}_{Q p}$, for a binary operator $\circ$ and a propositional quantifier $Q p$. In both cases, there are some independent reasons one might have for thinking that these principles are too strong.

In the case of $\mathrm{I}_{Q p}$, one might note that even if propositions reflect some of the logical structure of quantified sentences expressing them, it is plausible this does not include the order of quantifiers in any string of the same quantifiers, and it is plausible that the identity of propositions expressed is invariant under relabeling bound variables. Illustrating this using universal quantifiers, the following identifications are plausible:

$$
\begin{aligned}
& \text { (PERM) } \forall p \forall q \varphi=\forall q \forall p \varphi \\
& (\mathrm{VAR}) \forall q \forall p \varphi=\forall p \forall q(\varphi[p / q, q / p]) .
\end{aligned}
$$

Consider the instances of these principles for $\varphi$ being $p$. Together, they entail that $\forall p \forall q p=\forall p \forall q q$. But with $I_{\forall p}$, it follows that $\forall p \exists q(\forall q p=\forall q q)$. And any instance of this universal claim for a truth $p$ is false. Thus PERM and VAR are inconsistent with $\mathrm{I}_{\forall p}$.

These considerations may motivate restricting $\mathrm{I}_{Q p}$ to cases in which the complement clause $\varphi$ is a complex formula which does not itself start with a quantifier. But only such a restricted instance is appealed to in the results of Section 4 , so even this restricted version of $\mathrm{I}_{Q p}$, for a propositional quantifier $Q p$, is inconsistent with $\mathrm{O}_{\circ}^{-}$, for any binary sentential operator $\circ$.

In the case of binary sentential operators, recall how $\mathrm{O}_{\circ}$ was noted to be inconsistent with the commutativity of $\circ$, which motivated considering the weaker principle $\mathrm{O}_{\circ}^{-}$. As one might hold on to some version of the idea that propositions exhibit conjunctive and disjunctive structure while arguing that conjunction and disjunction are commutative, one might similarly hold on to this idea while arguing that conjunction and disjunction 
are associative. On this view, the following principle of associativity holds for both $\wedge$ and $\vee$ :

$$
(p \circ(q \circ r))=((p \circ q) \circ r) .
$$

Someone might want to endorse this even if they think that conjuncts may be recovered from conjunctions; on their view, there may simply be one conjunctive proposition with three conjuncts $p, q$ and $r$. As a commutative view of conjunction and disjunction can be illustrated by thinking of conjoining and disjoining propositions as akin to putting the conjuncts or disjuncts in a (conjunctive or disjunctive) bag, one can illustrate an associative view of conjunction and disjunction by thinking of conjoining and disjoining propositions as akin to gluing the conjuncts or disjuncts together (using a conjunctive or disjunctive glue).

The associativity of conjunction is inconsistent with $\mathrm{O}_{\wedge}^{-}$: If $(p \wedge(\neg p \wedge \neg p))=((p \wedge$ $\neg p) \wedge \neg p)$, then by $\mathrm{O}_{\wedge}^{-}, p$ must be $p \wedge \neg p$ or $\neg p$, which cannot be the case if $p$ is true. A similar argument shows that the associativity of disjunction is inconsistent with $\mathrm{O}_{\vee}^{-}$. Thus, one might argue that there are independent reasons for thinking that $\mathrm{O}_{\circ}^{-}$is too strong. On this view, the recovery of conjuncts and disjuncts has to be restricted to conjuncts and disjuncts which are not themselves conjunctions or disjunctions, respectively. That is, the relevant weakening of $\mathrm{O}_{\circ}^{-}$would be:

$$
\begin{aligned}
&\left(\mathrm{O}_{\circ}^{--}\right) \forall r\left(r=p \vee r=p^{\prime} \vee r=q \vee r=q^{\prime} \rightarrow \forall p \forall q(r \neq p \circ q)\right) \rightarrow \\
&\left(\left(p \circ p^{\prime}\right)=\left(q \circ q^{\prime}\right) \rightarrow\left(p=q \wedge p^{\prime}=q^{\prime}\right) \vee\left(p=q^{\prime} \wedge p^{\prime}=q\right)\right) .
\end{aligned}
$$

But as it turns out, even such a weakened principle is inconsistent with any principle $\mathrm{I}_{Q p}$, assuming a couple of natural auxiliary assumptions governing negation. The first is $\mathrm{O}_{\neg}$; the second is the following principle, stating that no negation is an application of $\circ$ :

$$
(\neg \neq \circ) \neg p \neq(q \circ r) .
$$

Proposition 36. If $Q p$ is a propositional quantifier and $\circ$ is a binary operator, then $\mathrm{O}_{\circ}^{--}$, $\mathrm{I}_{Q p}, \mathrm{O}_{\neg}$ and $\neg \neq \circ$ are jointly inconsistent.

Proof. Define:

$$
\varphi \text { o } \psi:=\neg(\neg(\neg \varphi \circ \neg \neg \varphi) \circ \neg(\neg \varphi \circ \neg \varphi)) \circ \neg(\neg \psi \circ \neg \psi) .
$$

Similar to the proof of Lemma 6, it can be shown that $\mathrm{O}_{\circ}^{--}, \mathrm{O}_{\neg}, \neg \neq \circ \vdash \mathrm{O}_{\circ}$. The claim follows along the lines of the proof of Lemma 7, with ô replaced by o.

There are various further weakenings which one might explore. For example, it might be argued that conjunction and disjunction are idempotent, so that $p \circ p=p$, for $\circ$ being $\wedge$ or $\vee$, without this trivializing the idea of conjunctive and disjunctive propositional structure. Such idempotence is again inconsistent with $\mathrm{O}_{\circ}^{-}$: by idempotence, $((p \circ \neg p) \circ(p \circ \neg p))=(p \circ \neg p)$, but by $\mathrm{O}_{\circ}^{-}$, it follows from this that both $p$ and $\neg p$ are $(p \circ \neg p)$. The weaker principle $\mathrm{O}_{\circ}^{--}$can therefore also be motivated by idempotence. Interestingly, in this case, one has independent reason to reject the auxiliary principle $\neg \neq \circ$ just appealed to, as the idempotence of $\circ$ immediately entails $\neg p=(\neg p \circ \neg p)$.

§7. Conclusion. Do propositions exhibit logical structure? Corollary 5 and Theorem 8 show, using the Russell-Myhill theorem, that there are significant logical 
limitations to any such logical structure: it cannot be that $p$ and $p p$ may always be recovered from $p \prec p p$, nor can it be, for any given binary sentential operator $\circ$ and propositional quantifier $Q p$, that $p$ and $q$ may always be recovered from $p \circ q$ and the instances may always be recovered from $Q p \varphi$.

If propositions exhibit logical structure, then they must do so in a more limited form. For example, it might be that the operands of sentential operators $\circ$ may be recoverable but not the instances of propositional quantifiers, or vice versa. Alternatively, it may be that both sentential operators and propositional quantifiers impart logical structure on the propositions expressed using them, but they do so in more restrictive ways. Section 6.2 provides some preliminary considerations in this direction, which indicate the existence of a large number of (combinations) of weaker principles of logical structure which might be explored.

Acknowledgements. For comments on drafts and presentations of this paper, I'd like to thank Andrew Bacon, Stephen Finlay, Simon Goldstein, Gabriel Uzquiano, Juhani Yli-Vakkuri, audiences at talks at ACU, University of Melbourne, and King's College London, and two anonymous referees for this journal. Special thanks to Jeremy Goodman and Cian Dorr for discussions which formed the basis of substantial parts of this paper: I owe the observation that the inconsistency result of Section 4 can be derived along the lines of the central result of [7] to Jeremy Goodman. The consistency of $\mathrm{I}_{\forall p}$ was first shown by Cian Dorr and Jeremy Goodman in correspondence, using a particular model. A version of this model can be found in Goodman [9]. The more abstract model constructions in Section 5 were obtained by generalizing and adapting this model.

\section{BIBLIOGRAPHY}

[1] Bealer, G. (1982). Quality and Concept. Oxford: Clarendon Press.

[2] Boolos, G. (1984). To be is to be a value of a variable (or to be some values of some variables). The Journal of Philosophy, 81, 430-449.

[3] Burgess, J. P., \& Rosen, G. (1997). A Subject with No Object. Oxford: Clarendon Press.

[4] Church, A. (1984). Russell's theory of identity of propositions. Philosophia Naturalis, 21, 513-522.

[5] Dorr, C. (2016). To be F is to be G. Philosophical Perspectives, 30, 39-134.

[6] Fine, K. (1970). Propositional quantifiers in modal logic. Theoria, 36, 336-346.

[7] Fritz, P. (n.d.). Ground and grain. Philosophy and Phenomenological Research, forthcoming.

[8] Fritz, P., Lederman, H., \& Uzquiano, G. (n.d.). Closed structure. Journal of Philosophical Logic, forthcoming.

[9] Goodman, J. (n.d.). Grounding generalizations. Unpublished.

[10] Hugly, P., \& Sayward, C. (1976). Prior on propositional identity. Analysis, 36, $182-184$.

[11] Myhill, J. (1958). Problems arising in the formalization of intensional logic. Logique et Analyse, 1, 78-83.

[12] Russell, B. (1903). The Principles of Mathematics. Cambridge: University Press.

[13] Stalnaker, R. (1977). Complex predicates. The Monist, 60, 327-339. 
[14] Uzquiano, G. (2019). Impredicativity and paradox. Thought, 8, 209-221.

[15] Walsh, S. (2016). Predicativity, the Russell-Myhill paradox, and Church's intensional logic. Journal of Philosophical Logic, 45, 277-326.

[16] Williamson, T. (1985). Converse relations. The Philosophical Review, 94, 249-262.

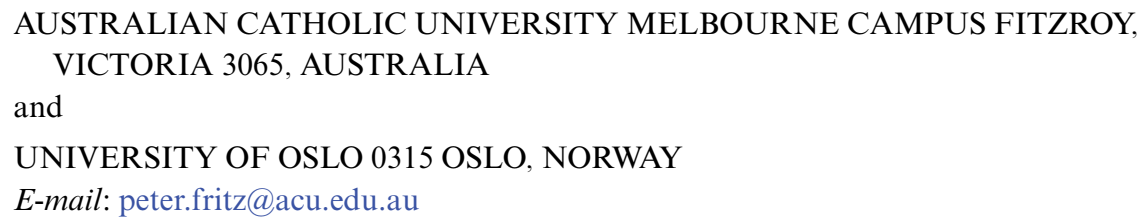

